Utah State University

DigitalCommons@USU

$2-2012$

\title{
The Effects of Surface Modification on Spacecraft Charging Parameters
}

Amberly Evans Jensen

Utah State University

JR Dennison

Utah State Univesity

Follow this and additional works at: https://digitalcommons.usu.edu/graduate_pubs

Part of the Physics Commons

\section{Recommended Citation}

Amberly Evans and JR Dennison, "The Effects of Surface Modification on Spacecraft Charging

Parameters," IEEE Trans. on Plasma Sci., 40(2), 305-310 (2012). DOI: 10.1109/TPS.2011.2179676

This Article is brought to you for free and open access by the Browse all Graduate Research at DigitalCommons@USU. It has been accepted for inclusion in Graduate Student Publications by an authorized administrator of DigitalCommons@USU. For more information, please contact

digitalcommons@usu.edu.

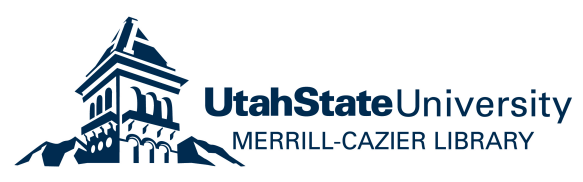




\title{
The Effects of Surface Modification on Spacecraft Charging Parameters
}

\author{
Amberly Evans and JR Dennison
}

\begin{abstract}
Charging of materials by incident radiation is affected by both environmental and physical conditions. Modifying a material's physical surface will change its reflection, transmission and absorption of the incident radiation which are integrally related to the accumulation of charge and energy deposition in the material. General arguments for incident and emitted photons, electrons and ions are considered. An optical analysis of the effects of surface modification on spacecraft charging parameters on prototypical polyimide Kapton $\mathrm{HN}^{\mathrm{TM}}$ and $\mathrm{Cu}$ samples is presented. Samples were roughened with abrasive compounds ranging from 0.5 to $10 \mu \mathrm{m}$ in size, comparable to the range of incident wavelengths. They were also contaminated with thin layers of DC 704 diffusion pump oil. Using a UV/VIS/NIR light source and a diffraction grating spectrometer, measurements were performed on pristine and modified materials. The measured spectra confirmed that surface modifications induce expected changes in optical reflection, transmission, and absorption. The generally increased absorption observed results in increased photon energy deposited in the material, leading to increased charge emission through the photoelectric effect.
\end{abstract}

Index Terms-Reflectivity, surface modification, spacecraft charging, photoyield, electron emission

\section{INTRODUCTION}

C HARGING of a material is affected by the physical conditions of the material [1,2], as well as environmental conditions [1,3]. Surface charging is typically limited to interaction of incident radiation in the outer $100 \mu \mathrm{m}$ of a material, as set by the range of incident and emitted particles. This limits the relevant incident energy of photons to $<10^{3} \mathrm{eV}$ (IR/VIS/VUV) [4,5], electrons to $<10^{5} \mathrm{eV}$ [6-9] and ions to $<10^{7}$ eV [9-11]; these energy ranges are the most intense spectral regions for typical space environments. Modifying a material's physical condition in this surface region will change its reflection, transmission, and absorption of the charge and energy of the incident radiation. Surface modifications include

Research was supported by funding from the NASA James Webb Space Telescope Program through Goddard Space Flight Center and a Utah State University Ecceles Research Fellowship and Undergraduate Research and Creative Opportunities grant.

Amberly Evans and JR Dennison are with the Materials Physics Group in the Physics Department at Utah State University in Logan, UT 84322 USA (e-mail: amb.eva@aggiemail.usu.edu, JR.Dennison@usu.edu).

Color versions of one or more figures in this paper are available online at http://ieeexplore.ieee.org.

Digital object identifier . roughening and deposition of thin film contaminant or overlayers. This study considers surface modifications to materials in a laboratory setting that simulate the effects of the space environment on the materials.

Simple considerations of conservation of charge and energy can be applied to the charging due to scattering of incident fluxes. An incident particle flux (photons, electrons or ions) produces reflected, transmitted and absorbed particle fluxes; energy can also be transferred to other particles and result in emitted fluxes of other species (see Figure 1). Each scenario for such general scattering can be described in terms of reflectivity $R$, transmissivity $T$, and absorptivity $A$, which are integrally related to the accumulation of charge and energy in a material; conservation requires that $R+A+T=1$. Perhaps the simplest scattering case to consider —-with the same incident and scattered species-is for incident photons with reflected and transmitted light and absorbed energy (see Figure 2). An analogous example with incident, reflected, transmitted and absorbed electrons is conventionally described in terms of secondary and backscattered yields and accumulated charge $[12,13]$. More complex examples of hybrid scattering with different incident and emitted particles that also affect spacecraft charging include: photoemission (photons in, electrons out) $[1,14]$; luminescence (photons or electrons in, photons out) $[1,15,16]$; and ion yields (ions in, electrons out) [1]. The familiar theoretical expressions for the reflectivity of photons from modified surfaces developed in Section II and corresponding experimental results presented in Section III have parallel developments for these more complex scattering scenarios.

Consider now the cases for incident photons with emitted photons (light scatter), electrons (photoyield) or ions

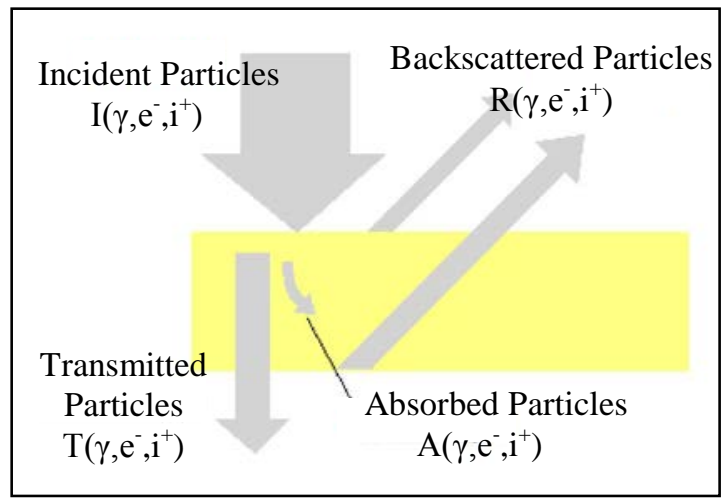

Figure 1. General scattering of incident particles. 
(ablation). Incident photons do not deposit charge, but do impart energy to the material. Increased photon absorption means increased photon energy is deposited in the material, which can lead to increased charge emission-and concomitant charging-through the photoelectric effect. (Ion emission has similar charging consequences, but is much less prevalent, due to high ion mass and low photon momentum.) Surface modification from increased surface roughness affects photon emission (optical reflection) or electron emission (secondary and backscattered yields) in various ways. Very shallow surface relief can increase particle emission by: (i) increasing the emitting area and (ii) causing more grazing incidence, resulting in energy deposition nearer the surface. By contrast, deeper roughening [on the order of the secondary electron mean free path $(\sim 1 \mathrm{~nm})$ or larger] can reduce electron emission and enhance negative charging by reabsorbing emitted electrons. As an extreme example, a material comprised of very deep features with very thin walls acts essentially as a Faraday cup, effectively trapping all emitted photons or electrons so that total electron yield approaches zero and net positive charging can no longer occur. Surface modification from contamination involves depositing a foreign substance on the surface of the material. It is expected that as the thickness of the layer of foreign substance increases, the optical properties will be increasingly different from the uncontaminated material and ultimately approach those of the contamination. Thus, modifying the surface of a material consequently affects the photon-induced charging of the material.

\section{THEORY}

The photoelectric effect is one way in which spacecraft build up charge. Absorbed incident photons will deposit energy in the material, but photons that are reflected or transmitted do not deposit energy. It is possible, though, for reflected photons to be reabsorbed and then contribute to the total deposited energy in the material. Standard optical theories of light scattering have been developed to predict reflection, transmission and absorption from materials properties for interfaces, rough surfaces, thins films, coated thin films and multilayer thin films [17-19].

Upon contact with the material, light is reflected from the top surface or enters the material. That light which enters either transmits all the way through, reflects off the bottom surface or is absorbed in the sample (Figure 2(a)). From the Fresnel equations, reflectance, $R$, at normal incidence from a material of index of refraction $n_{0}$ into a material of index $n_{1}$ is [17]:

$$
R=\left[\frac{n_{1}-n_{0}}{n_{1}+n_{0}}\right]^{2}
$$

When light is incident on a roughened material, scattering can change (Figure 2(b)). The uneven surface scatters the reflected light diffusely in all directions. The 'valleys' created by roughening, 'trap' some of the reflected light which can be reabsorbed by the material. The reflectivity due to diffuse

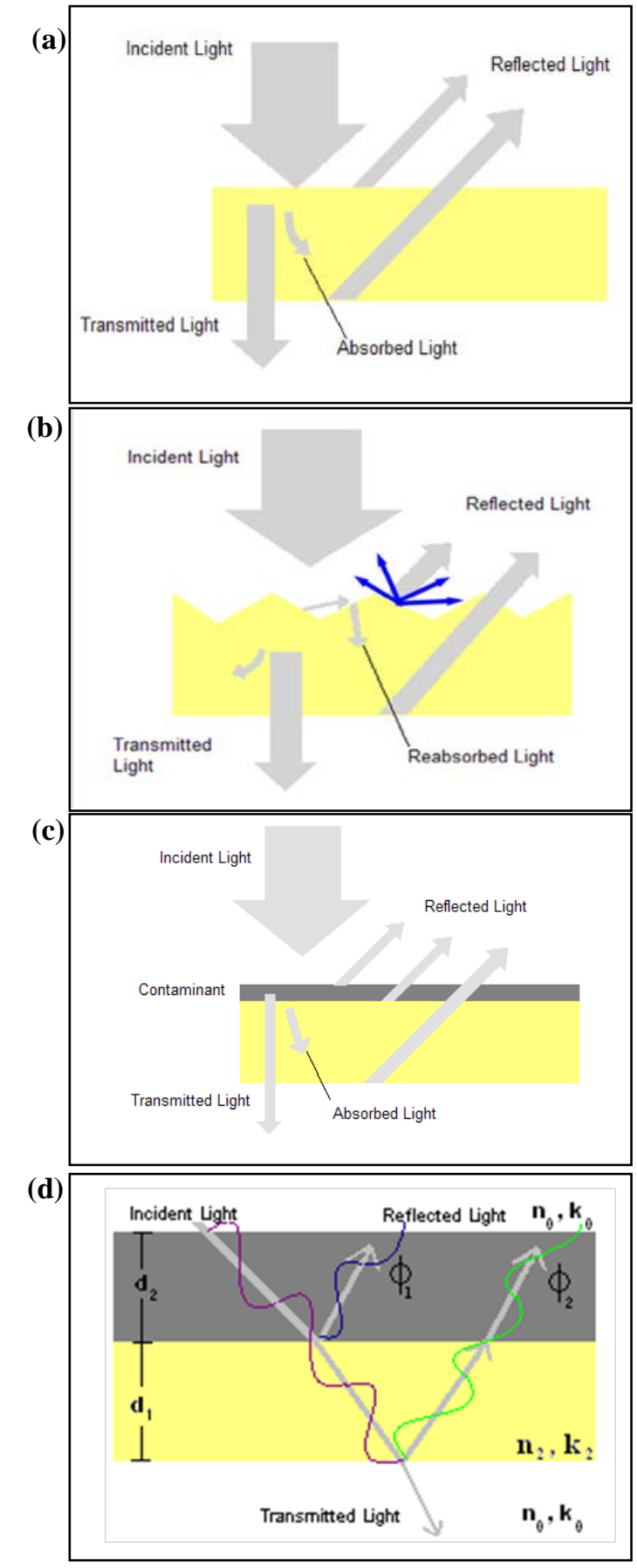

Figure 2. Scattering of incident light for (a) pristine material, (b) roughened material, (c) material with thin film contamination layer and (d) constructive and destructive interference from multiple layers.

reflectance, $R_{\text {diff, }}$ can be expressed as [18]

$$
R_{\text {diff }}=(1-\Delta) R
$$

where $\Delta$ is the fraction of light reabsorbed.

Contamination of a material by a thin layer creates a third reflecting surface for incident light, changing the overall reflection (Figure 2(c)). Light can now also be absorbed within the contaminant layer. The relation between the absorption coefficient $[17,18]$ and the reflectivity for the contaminant layer of thickness $x$ is 


$$
R_{\text {Cont }}(x)=e^{-2 \alpha x} \quad \text { or } \quad \alpha=-\ln \left(R_{\text {Cont }}\right) / 2 x
$$

The combined multilayer reflectivity due to these three reflecting surfaces of thickness $d_{i}$ (e.g., the three upward arrows in Figure 2(c)) is [18,19]:

$$
\begin{aligned}
R_{M L}=\left[\frac{n_{1}-n_{0}}{n_{1}+n_{0}}\right]^{2}+\left[\frac{2 \sqrt{n_{1} n_{0}}}{n_{1}+n_{0}}\right]^{2}\left[\frac{n_{2}-n_{1}}{n_{2}+n_{1}}\right]^{2} e^{-2 \alpha_{1} d_{1}} \\
+\left[\frac{2 n_{1} n_{0}}{n_{1}+n_{0}}\right]^{2}\left[\frac{2 n_{2} n_{1}}{n_{2}+n_{1}}\right]^{2}\left[\frac{n_{0}-n_{2}}{n_{0}+n_{2}}\right]^{2} e^{-2 \alpha_{1} d_{1}} e^{-2 \alpha_{2} d_{2}}
\end{aligned}
$$

The first term on the right hand side of the equation represents reflected light from the first surface reflection [from Eq. (1)]. The second term is the product of the transmission through the first surface times the reflection from the second surface [from Eq. (1)] reduced by the exponential factor for the absorption passing through the contaminant layer twice [from Eq. (2)]. The third term represents product of the transmission through the first and second surfaces twice each, times the reflection from the bottom surface, with light reduced by absorption passing through the both the contaminant layer and the substrate twice [This ignores multiple reflections.].

Reflected light from each layer can combine, leading to constructive or destructive interference at different wavelengths and causing thin film interference patterns in the reflectance versus wavelength curves. Figure 2(d) illustrates this effect. From the thin film interference patterns, the index of refraction can be calculated from the spacing of two successive interference maxima of the wavelength spectrum as $[17,18]$

$$
n=\frac{1}{2 d} \frac{\lambda_{2} \cdot \lambda_{1}}{\lambda_{2}-\lambda_{1}}
$$

The total reflectance, including interference, for a given incident wavelength is [17]

$$
R_{\mathrm{int}}=\frac{1}{2}\left[\frac{n_{1}-n_{0}}{n_{1}+n_{0}}\right]^{2} \cdot\left\{\begin{array}{ll}
\left(1+e^{-4 \alpha d}\right) & ; \text { incoherent }, \\
\left(1+e^{-2 \alpha d}\right)^{2} & ; \text { coherent, constructive } \\
\left(1-e^{-2 \alpha d}\right)^{2} & ; \text { coherent, destructive }
\end{array}\right\}(6)
$$

\section{EXPERIMENTAL METHODS}

Two representative materials, polyimide Kapton $\mathrm{HN}^{\mathrm{TM}}$ and oxygen free high-conductivity (OFHC) copper, were analyzed. A pristine sample of each was used as a control. Four samples of $27 \mu \mathrm{m}$ thick Kapton HNTM, a ubiquitous thin film insulating material, were prepared from as-received material. Five samples of OFHC $\mathrm{Cu}$ were prepared by polishing the surface, using decreasing sizes of polishing compounds, down to $1 / 4 \mu \mathrm{m}$, so that the surface was mirrorlike. Four samples of $\mathrm{Cu}$ and two of polyimide were then roughened, each with a different uniform size of alumina polishing compound, creating different sizes of scratches in each sample (1, 3, 6 and $9.5 \mu \mathrm{m}$ for $\mathrm{Cu}$ and 1 and $9.5 \mu \mathrm{m}$ for

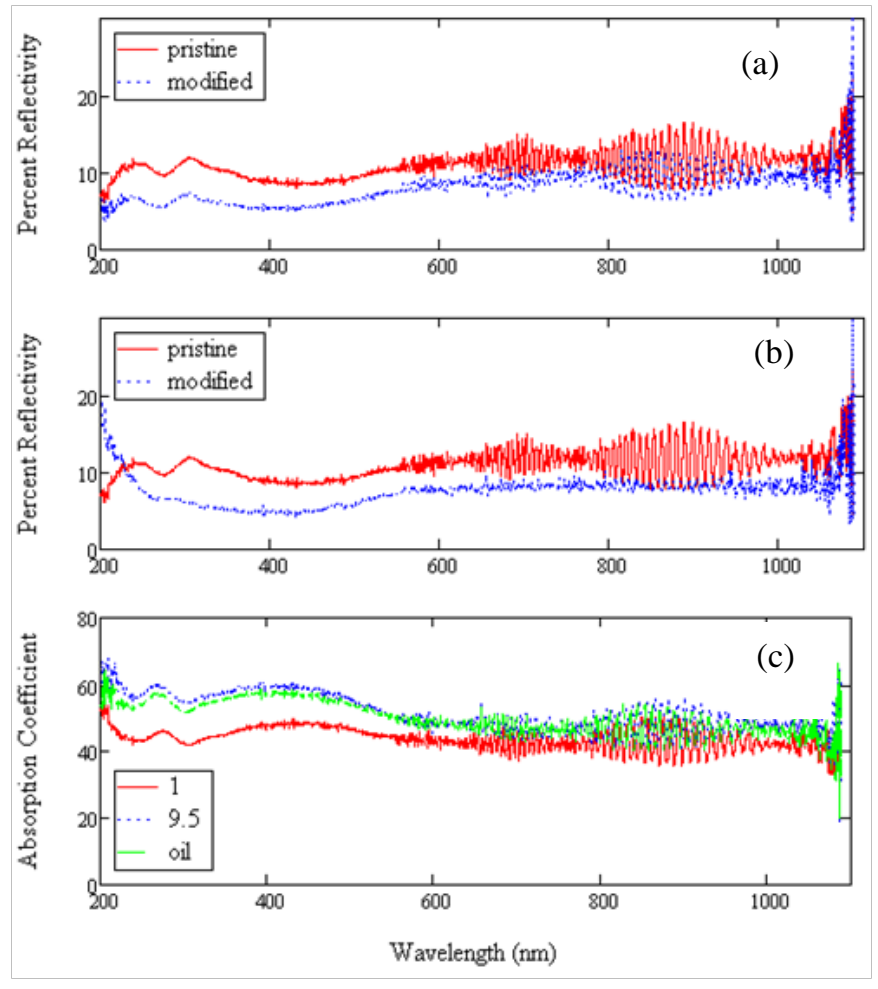

Figure 3. Specular reflectivity of $27 \mu \mathrm{m}$ thin film polyimide Kapton HN ${ }^{\mathrm{TM}}$ samples. (a) Pristine sample (red) and sample roughened with $9.5 \mu \mathrm{m}$ particles (blue). (b) Pristine sample (red) and a sample with a thin layer of DC 704 diffusion pump oil contaminant (blue). (c) Absorption coefficient as a function of wavelength for modified samples; (red) roughened with $1 \mu \mathrm{m}$ particles; (blue) roughened with $9.5 \mu \mathrm{m}$ particles and (green) contaminated with a thin layer of DC 704 diffusion pump oil.

polyimide). A fourth polyimide sample was prepared with a thin contaminant film. Dow Corning DC 704 diffusion pump oil (tetramethyltetra-phenyltrisiloxane)—with $\mathrm{n}=1.50$ —was used to mimic common spacecraft organic contaminants $[13,20]$.

Using a UV/VIS/NIR tungsten halogen light source (200 $\mathrm{nm}$ to $1100 \mathrm{~nm}$ ) and diffraction grating spectrometer (Ocean Optics, Model HR4000), normal specular and diffuse optical reflectivity measurements were made. Specular reflection was obtained using a fiber optic probe that was positioned perpendicular to the sample, which measured a surface area of $\sim 400 \mu \mathrm{m}$ diameter. An integrating sphere was used to measure the diffuse reflection. Note that at wavelengths below $\sim 300 \mathrm{~nm}$ and above $\sim 1050 \mathrm{~nm}$, intensity ratios are sometimes inaccurate due to low scattering and detector efficiencies.

\section{RESUltS}

The results of Kapton HNTM reflectivity measurements are shown in Fig. 3. Figure 3(a) shows the difference between the pristine sample and that roughened with $9.5 \mu \mathrm{m}$ particles. The average reflectivity is reduced to $\sim 9.5 \%$ by roughening over the full range of wavelengths. Thin film interference patterns are still observed even with roughening. The magnitude of the interference fringes observed for these films estimated using Eq. (6) is consistent with the average absorption coefficient in the infrared region (see Fig. 3(c)). These reflectance 

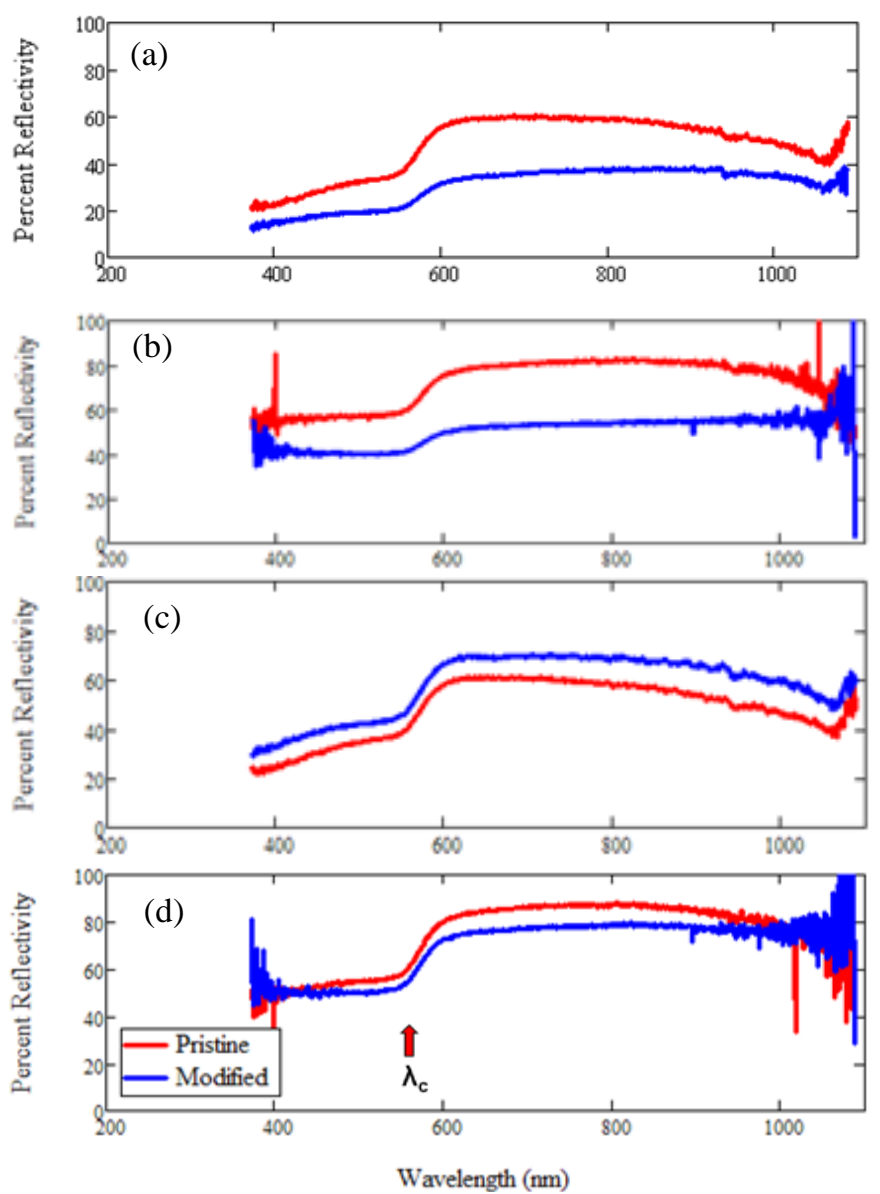

Figure 4. (a) Normal specular reflectance and (b) diffuse reflectance of $\mathrm{Cu}$; pristine (red), roughened with $9.5 \mu \mathrm{m}$ (blue). (c) Normal specular reflectance and (d) diffuse reflectance of $\mathrm{Cu}$; pristine (red) and roughened with $1 \mu \mathrm{m}$ (blue) abrasive particles.

measurements were taken multiple times at different locations on the samples. The behavior of the spectra is not consistent from one measurement to the next, suggesting that different areas of the roughened sample scatter differently; this is consistent with inhomogeneous roughening that would be expected from a polishing process.

The average reflectance is reduced to $\sim 8 \%$ by the DC-704 contamination (see Figure 3(b)). This is consistent with a reduction based on Eq. (1) for $n=1.35$ for DC-704, in good agreement with the manufacturer's value [21]. Thin film interference patterns are almost fully damped, suggesting a loss of coherence in the scattered light. At very low wavelengths, the reflectivity of the contaminated sample increases, as a result of higher reflectivity of the contaminate oil in this spectral region [22].

For all but one of the roughened $\mathrm{Cu}$ samples, we observe a reduction in reflectivity for both normal specular (Figures 4(a) and 4(c)) and diffuse (Figures 4(b) and 4(d)) reflection. The cutoff wavelength, $\lambda_{c}$, for $\mathrm{Cu}$ at $2.12 \mathrm{eV}$ is indicated on the plots; this dramatic increase in absorption results from $3 \mathrm{~d}$ to $4 \mathrm{~s}$ band transitions where flat dispersion relations lead to high concentrations in the densities of state and thereby large transition probabilities [23]. In normal specular reflectance for samples roughened with $9.5 \mu \mathrm{m}$ particles, the decrease is 8-12\% below $\lambda_{c}$ and $12-30 \%$ above $\lambda_{c}$. Likewise, diffuse
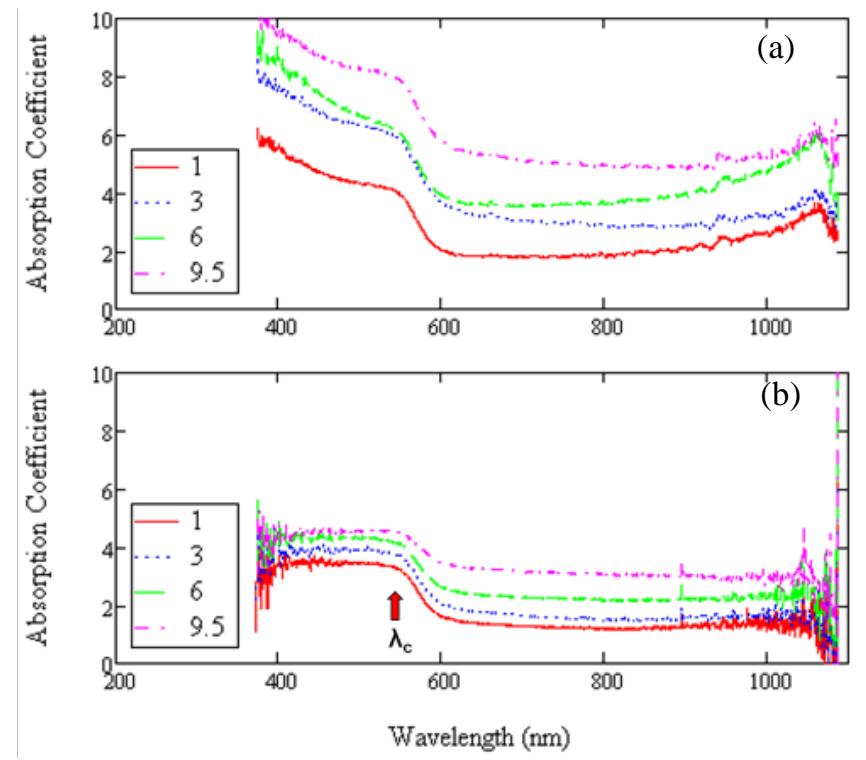

Figure 5. Absorption coefficient as a function of wavelength for samples of $\mathrm{Cu}$ roughened with $1 \mu \mathrm{m}$ (red), $3 \mu \mathrm{m}$ (blue), $6 \mu \mathrm{m}$ (green) and $9 \mu \mathrm{m}$ (magenta) abrasive compounds. (a) Normal specular reflectance. (b) Diffuse reflectance.

reflectance decreases $12-20 \%$ below $\lambda_{c}$ and $20-30 \%$ above $\lambda_{c}$.

By contrast, roughening with $1 \mu \mathrm{m}$ particles (Fig. 4(c)) actually increased normal specular reflectance $5-10 \%$ below $\lambda_{c}$ and $0-3 \%$ above $\lambda_{c}$. This increase in reflectance is attributed to an increase in reflecting area due to the small scratches that are not deep enough to enhance reabsorption of light. Diffuse reflectance (Fig. 4(d)) for this sample decreases only 2-8\% below $\lambda_{c}$ and $5-10 \%$ above. $\lambda_{c}$

\section{CONCLUSION}

The purpose of these measurements was to ultimately learn about the effect that these surface modifications have on charging. From the reflectivity, we can obtain absorptivity information. Absorptivity will finally lead to photoyield information.

The absorption coefficients for each modification of Kapton HN $^{\text {TM }}$ have been calculated using Eq. (3) and are plotted as a function of wavelength in Fig. 3(c). The absorption coefficients increase as roughening size increases and when a contaminant layer is deposited on the surface. This demonstrates that absorptivity increases upon roughening and-for DC 704 diffusion pump oil—upon contamination.

For the $\mathrm{Cu}$ samples, the absorption coefficient was calculated for both the normal specular and diffuse reflectance (Figures 5(a) and 5(b), respectively). As roughening size increases, the absorption coefficient also increases. This is as expected. Increased absorption indicates that charging is increased through the photoelectric effect. Also calculated was the average percent change in reflectivity, $\Delta$, from Eq. (2). For both normal specular and diffuse reflectance, the relation between $\Delta$ and roughening particle size is linear (Fig. 6). The rate of change for reflectance is $\sim 35 \%$ larger for specular than diffuse scattering; this is consistent with high angle diffuse scattering from depressed surface areas being recaptured by adjacent elevated areas. 


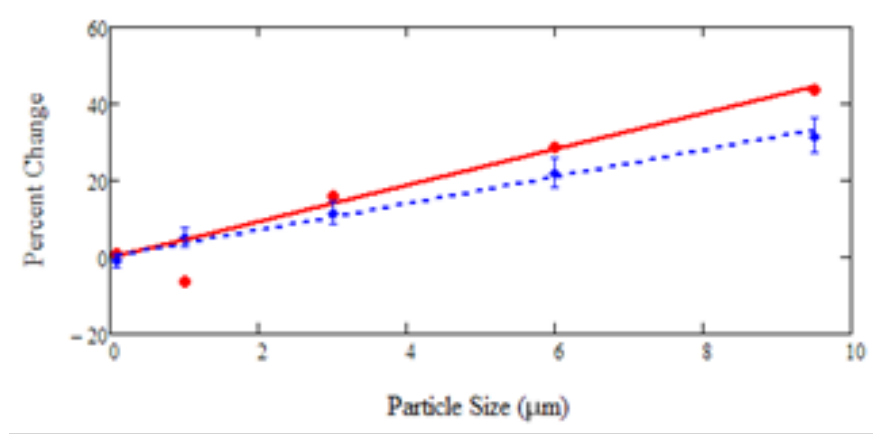

Figure 6. Average percent decrease in reflectivity, $\Delta$, versus roughening particle size for normal specular (red circles) and diffuse (blue diamonds) reflectance of $\mathrm{Cu}$ samples. Linear fits to the data are shown, with $(0.47 \pm 0.06)$ and $(0.35 \pm 0.03) \%$ change per $\mu \mathrm{m}$ roughening, for specular and diffuse reflectivity, respectively.

For spacecraft charging applications, the effects on the total photoyield, $\sigma_{P h}$, of changes in reflectivity through related changes in absorbtivity often have the most pronounced effects $[1,14]$. Based on arguments outlined by Lai [14] and Dennison [1],

$$
\begin{aligned}
\sigma_{P h}=\frac{1}{N_{e}^{T o t}} \int & {\left[\frac{d N_{P h}\left(E_{P h}\right)}{d E_{P h}} \cos (\varphi)\right]\left[\frac{\sigma_{P h}^{n}\left(E_{P h}\right)}{\cos (\varphi)}\right] } \\
& \left.\times\left\{1-R\left(E_{P h}\right)-T\left(E_{P h}\right)\right] \cos (\varphi)\right\} d E_{P h}
\end{aligned}
$$

The right hand expression for photoyield is normalized by the total number of incident electrons, $N_{e}^{T o t}$. The first factor in the integral scales the differential number of photoelectron with respect to photon energy, $d N_{P h} / d E_{P h}$, to account for the decrease in cross sectional area with angle of incidence, $\varphi$. The second factor is the enhancement of the differential photoyield for normal incidence, $\sigma_{P h}{ }^{n}\left(E_{P h}\right)$, due to photon penetration depth. The last factor in curly brackets is the optical absorptivity that corrects $\sigma_{P h}{ }^{n}$ for the fact that only absorbed photons deposit energy in the material and can thereby produce photoelectrons (From the simple conservation arguments in the Introduction, this absorptivity is equal to one minus the sum of the reflectivity plus transmission at normal incidence.), all scaled by a Lambertian $\cos (\varphi)$ factor [1]. The inherent absorptivity and transmissivity at normal incidence are complex properties of the microscopic bandstructure and macroscopic dielectric properties of the material and can depend heavily on the incident photon energy, $E_{P h}$. These can be modified by structural changes in the material, or through UV or radiation damage. As discussed above, reflectivity at normal incidence depends more critically on surface modifications (on the order of incident optical wavelengths), through changes in surface roughness, contamination, surface degradation (e.g., from atomic oxygen), or temperature. Changes in surface material absorptivity and emissivity can also modify the thermal response of a satellite and thereby the temperature of the spacecraft materials. Materials properties related to charging-including conductivity, radiation induced conductivity, dielectric constant, electrostatic breakdown strength, and electron yields-are often temperature

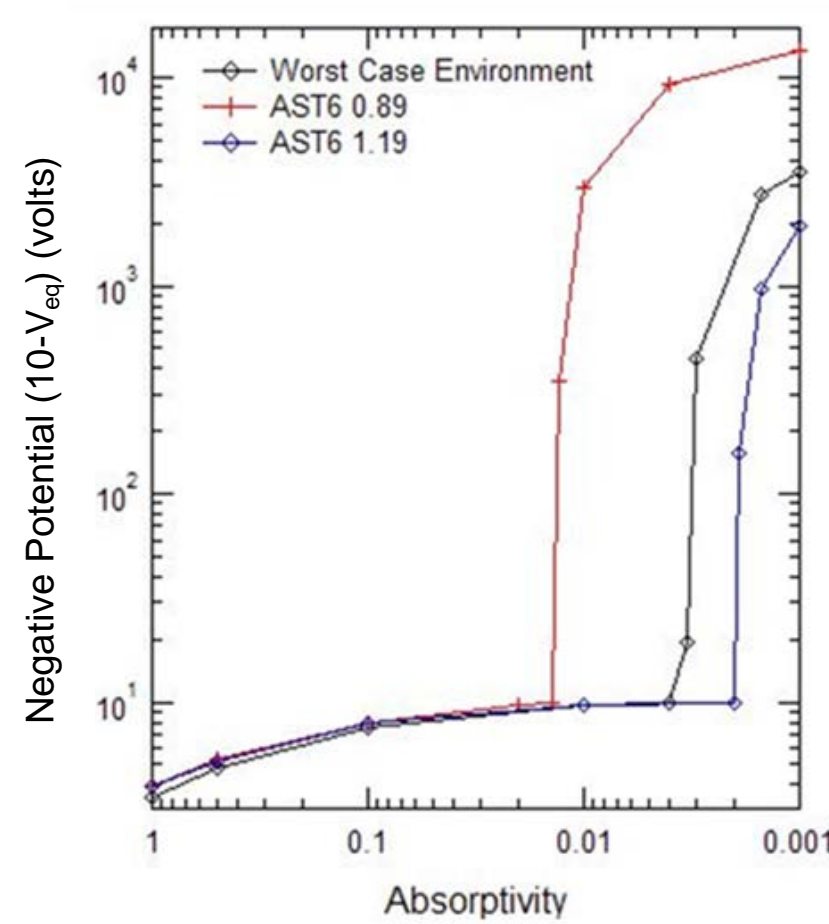

Figure 7. Equilibrium charging potential for a flat, twodimensional satellite panel of $\mathrm{Au}$ as the fraction of absorbed photon energy decreases from $100 \%$ to $0 \%$. Curves are for the 4 September 1997 (squares), worst case (circles), and ATS-6 (triangles) geosynchronous environments [1].

dependant, especially for polymeric insulators [24]. Thus, optical absorptivity can have indirect effects on charging, in addition to those from photoyield.

Under suitable circumstances, an increase in optical absorptivity can lead to threshold charging at finite values. Figure 7 shows one such example, the calculated equilibrium potential of a flat panel of $\mathrm{Au}$ for three specific conditions. In full sunlight these panels exhibit positive charging [1]. However, as the absorbtivity is reduce below from $2 \%$ to $0.2 \%$ (depending on specific conditions), the panel undergoes threshold charging. These results confirm the predictions of Lai, who calculated the critical temperature as a function of the reflectivity for several materials [1,14].

The measurements and calculations for incident and reflected light described in detail above can serve as a model for scattering related to spacecraft charging other than photon in-photon out processes. Specifically, we have begun to apply reflection/transmission/absorption notions to model the effects of surface roughening, as well as contamination and multilayer materials, for the case of incident and scattered electrons. This includes models of backscattered electrons and secondary electrons [20], such as the Chung and Everhardt model for secondary electron emission spectra [12] and the effects of charge accumulation on electron emission [25].

\section{REFERENCES}

[1] JR Dennison, R.C. Hoffmann, and J. Abbott, "Triggering Threshold Spacecraft Charging with Changes in Electron Emission from Materials," Paper AIAA-2007-1098, Proceedings of the $45^{\text {th }}$ American Institute of Aeronautics and Astronomics Meeting on Aerospace Sciences, Reno, NV, January, 10, 2007. 
[2] JR Dennison and RC Hoffmann, "Effects on Spacecraft Charging of Modification of Materials by Space Environment Interactions," submitted to IEEE Transaction on Plasma Science, 2011.

[3] D. Hastings, H. Garrett, Spacecraft-Environment Interactions, New York, NY: Cambridge Press, 1996.

[4] Bohren,C. F. and Huffman, D.R. "Absorption and Scattering of Light by Small Particles", Wiley, (1983).

[5] M.J. Berger, J.H. Hubbell, S.M. Seltzer, J. Chang, J.S. Coursey, R. Sukumar, D.S. Zucker, and K. Olsen, XCOM: Photon Cross Sections Database, NIST Standard Reference Database 8 (XGAM), NBSIR 873597, NIST, Gaithersburg, 2010. http://www.nist.gov/pml/data/xcom/index.cfm.

[6] G. Wilson and JR Dennison, "Approximation of Range in Materials as a Function of Incident Electron Energy," submitted to IEEE Transaction on Plasma Science, 2011.

[7] ICRU (1984). International Commission on Radiation Units and Measurements. ICRU Report 37, Stopping Powers for Electrons and Positrons.

[8] National Institute of Standards and Technology, 2010, "ESTAR, Stopping Power and Range Tables for Electrons" (http://physics.nist.gov/PhysRefData/Star/Text/ESTAR.html).

[9] Bethe, H. A., "Zur Theorie des Durchgangs schneller Korpuskularstrahlen durch Materie,” Ann. d. Physik 5, 325, 1930.

[10] ICRU (1993). International Commission on Radiation Units and Measurements. ICRU Report 49, Stopping Powers and Ranges for Protons and Alpha Particles.

[11] National Institute of Standards and Technology, 2010, "PSTAR, Stopping Power and Range Tables for Protons" (http://physics.nist.gov/PhysRefData/Star/Text/PSTAR.html).

[12] M. Chung and T. Everhart, "Simple calculation of energy distribution of low energy secondary electrons emitted from metals under electron bombardment," J. of Appl. Phys., vol. 45, p. 707, 1974.

[13] Davies, R.E., and J.R. Dennison, Evolution of secondary electron emission characteristics of spacecraft surfaces, J. Spacecraft and Rockets, Vol. 34, No. 4, 1997, pp. 571-574.

[14] Lai, S.T. and Tautz, M., "Aspects of Spacecraft Charging in Sunlight," IEEE Transaction on Plasma Science, Vol. 34, No. 5, October, 2006, pp. 2053-2061.

[15] V Griseri, L A Dissado, J C Fothergill, C Laurent and G Teyssèdre, "Photoluminescence, recombination induced luminescence and electroluminescence in epoxy resin,” J. Phys. D: Appl. Phys. 34 2534, 2001.

[16] J.A. Roth, R. Hoffmann, J.R. Dennison, and J. R. Tippetts, "Effects of Radiation Induced Conductivity on Electrostatic Discharge in Insulating Materials,” Paper Number: AIAA-2009-3527, Proc. of 1st AIAA Atmospheric and Space Environ. Conf., 2009.

[17] Hecht, Eugene, Optics, $3^{\text {rd }}$ Ed., Reading, MA: Addison-Wesley, 1998.

[18] O.S. Heavens, Optical Properties of Thin Solid Films, London: Butterworth Scientific, 1955.

[19] Jena, K.C., and D.K. Hore, "A Simple Transmission-based Approach for Determining the Thickness of Transparent Films," Am. J. Phys., 79, 256-260 (2011).
[20] Chang, W.Y., Dennison, J.R., Kite, Jason and Davies, R.E., "Effects of Evolving Surface Contamination on Spacecraft Charging," Proceedings of the $38^{\text {th }}$ AIAA Meeting on Aerospace Sciences, Reno, NV, 2000.

[21] "Product Information: Dow Corning 704 Diffusion Pump Oil," Document 10-838-98, Dow Corning Corp., Corning, NY, 1998.

[22] J.M. Maloney, "The Comparison of Absorbance Infrared Radiation Spectra of Thin Oil Films on Diffuse and Specular Surfaces," MS Thesis, Logan, UT: Utah State University, 1996.

[23] N.W. Ashcroft and N.D. Mermin, Solid State Physics, New York, NY: Holt, Rinehart and Winston, 1976.

[24] JR Dennison and RC Hoffmann, "Effects on Spacecraft Charging of Modification of Materials by Space Environment Interactions," 11th Spacecraft Charging Technology Conference, (Albuquerque, NM, September 20-24, 2010).

[25] Hoffmann, RC and JR Dennison, "Methods to Determine Total Electron-Induced Electron Yields Over Broad Range of Conductive and Nonconductive Materials," submitted to IEEE Transaction on Plasma Science, 2011.

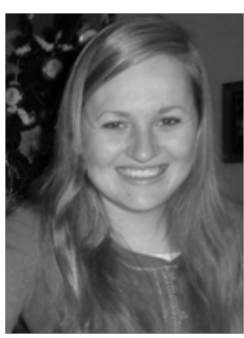

Amberly Evans is a senior dual physics and chemistry major at Utah State University in Logan, UT. She has worked with the Materials Physics Group for four years on electron emission, luminescence and resistivity studies and on MISSE retrieval and post-flight analysis of SUSpECS. Much of her work has focused on optical scattering of spacecraft materials.

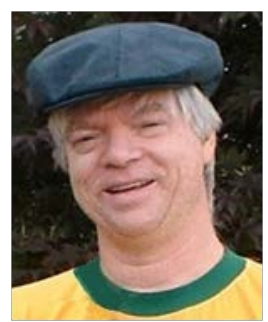

J. R. Dennison received the B.S. degree in physics from Appalachian State University, Boone, NC, in 1980, and the M.S. and Ph.D. degrees in physics from Virginia Tech in Blacksburg, in 1983 and 1985, respectively. He was a Research Associate with the University of Missouri-Columbia before moving to Utah State University (USU), Logan, in 1988. He is currently a Professor of physics with USU where he leads the Materials Physics Group. He has worked in the area of electron scattering for his entire career and has focused on the electron emission and resistivity of materials related to spacecraft charging for the last two decades. 